\title{
Politiques de décentralisation en éducation : diversité internationale, légitimations théoriques et justifications empiriques
}

Nathalie Mons

Depuis deux décennies, les réformes de décentralisation de l'éducation se sont multipliées sur tous les continents. De nombreux pays, aux caractéristiques économiques, démographiques ou éducatives très diverses, ont mis en œuvre ces politiques éducatives multiformes. Multiples par nature, elles ont fait exploser l'opposition schématique entre États centralisés et États fédéraux : la typologie de la répartition des compétences en éducation s'est enrichie de nouvelles familles. Pourtant, l'engouement pour la gouvernance locale ne s'appuie pas sur un corpus théorique solide, pas plus qu'il ne trouve sa légitimité dans la recherche empirique. Le débat sur les conséquences scolaires de la décentralisation de l'éducation n'est, à ce jour, pas clos.

Mots-clés : décentralisation, autonomie scolaire, performance scolaire, éducation comparée, évaluation.

\section{INTRODUCTION}

"Lorsque les responsables politiques et les analystes tirent des leçons de la littérature actuelle sur la décentralisation, ils doivent être conscients que les arguments appuyant ce type de réformes tiennent plus d'espoirs et de préférences personnelles que de résultats empiriques fondés. La décentralisation en éducation... conduit à un processus turbulent et complexe, dont l'évolution et les résultats ne sont souvent ni explicables, ni prévisibles. »

C'est en ces termes crus que la chercheuse de I'Université de Stanford, Diana Rothen (2000), pointe du doigt les carences qui caractérisent aujourd'hui les analyses et les politiques de décentralisation de l'éducation.

II est vrai qu'en France comme à l'étranger, la décentralisation est au cœur des débats nationaux, les politiques opèrent de nombreux revirements, le chemin emprunté par les réformes est hasardeux. Ainsi, la Hongrie qui avait fait le choix d'une très forte décentralisation au début des années 90 est revenue en arrière en 1998, les pouvoirs du gouvernement central ont été renforcés. De même, le Royaume-Uni, adepte de l'ultra-décentralisation, a adopté sous le gouvernement Blair les "stratégies de lecture et de 
mathématiques ", qui prévoient désormais pour le primaire et le secondaire un encadrement des méthodes pédagogiques très contraignant. Des États pourtant très attachés au fédéralisme, comme les États-Unis ou l'Australie, ont également mis en place, depuis la fin des années 90, des standards pédagogiques nationaux qui limitent la marge de manœuvre de leurs États.

Ces revirements de politiques révèlent un phénomène : chercheurs et responsables politiques s'interrogent sur la meilleure répartition des compétences en éducation. Le modèle longtemps prédominant de l'État centralisé est aujourd'hui battu en brèche. L'État-providence a déçu, la planification centralisée n'a pas permis le développement économique tant espéré ni la qualité de l'enseignement tant attendue. Surtout, la massification de l'éducation semble rendre les structures centrales lourdes à gouverner.

Les réformes de décentralisation se sont donc multipliées dans les années 80 et accélérées dans les années 90 . Elles ont transcendé les clivages économiques, spatiaux et politiques pour devenir des "politiques supra-nationales ». Multiples par nature, comme nous le verrons dans une première partie, elles font exploser l'opposition schématique entre États centralisés et États fédéraux : la typologie de la répartition des compétences en éducation s'est enrichie de nouvelles familles (seconde partie).

Dans une troisième partie, nous verrons cependant que l'engouement pour la gouvernance locale ne s'appuie pas sur un corpus théorique solide, pas plus qu'il ne trouve sa légitimité dans la recherche empirique (quatrième partie).

\section{LA DÉCENTRALISATION : UN PHÉNOMĖNE AUX VISAGES MULTIPLES}

Si la décentralisation apparaît comme un des principaux instruments de politique éducative des deux dernières décennies, c'est parce que le terme englobe, en fait, une multitude de réformes éducatives. Elles ont toutes pour objectif d'alléger les prérogatives de l'État central dans la gestion des systèmes éducatifs, en les transférant vers des acteurs locaux.

La littérature distingue en général trois formes de décentralisation (Rondinelli, Nellis et Cheema, 1984) qui diffèrent principalement par le degré d'autonomie des unités sub-nationales par rapport au gouvernement central :

a) la "déconcentration " dans laquelle le transfert des compétences se fait au profit d'unités locales qui restent sous l'autorité du gouvernement central (les académies en France, par exemple) ;

b) la " délégation ", c'est-à-dire le transfert de responsabilités à des unités sub-nationales ou des unités publiques, qui n'appartiennent pas au ministère de l'Éducation mais dont les pouvoirs sont clairement encadrés par l'autorité centrale (par exemple, les municipalités danoises interviennent en matière d'éducation (financement, pédagogie...) dans un cadre défini par l'État central) ;

c) la « dévolution ", par laquelle le transfert des responsabilités se fait en faveur d'unités sub-nationales et/ou publiques, indépendantes du ministère de l'éducation et bénéficiant d'une large autonomie de décision, accordée souvent de façon pérenne. En Espagne, par exemple, de par la constitution, les provinces autonomes sont les autorités en charge de l'éducation.

Si Rondinelli n'a considéré que des entités publiques, cet article appréhendera plus largement le phénomène de la décentralisation et prendra également en considération le transfert de compétences vers des unités sub-nationales privées. On rejoint ainsi Bennet (1990) qui distingue deux formes de décentralisation : la " décentralisation inter-gouvernementale » et la « décentralisation-privatisation ».

Donc, de façon synthétique, par décentralisation, nous entendrons ici le transfert de responsabilités, en matière de planification, management, financement et allocation des ressources, du gouvernement central vers des unités locales sub-nationales publiques ou privées, ces unités pouvant soit être directement placées sous l'autorité du gouvernement, soit disposer d'une autonomie partielle ou totale.

En conséquence, le champ d'investigation de l'article est large. Il couvre à la fois les déconcentrations (Portugal, Corée, Thaïlande...), les phénomènes de "provincialisation » ou " municipalisation » dans lesquels les collectivités locales se voient confier de nouvelles responsabilités éducatives (Danemark, Espagne ou Suède) ou encore les expériences d'autonomie scolaire (Nouvelle-Zélande, Royaume-Uni ou Hongrie). Les politiques qui favorisent le développement de réseaux d'enseignement alternatifs, pour remédier à ce qui est perçu comme des carences de l'instruction traditionnelle publique, ne seront pas oubliées. Ces réseaux peuvent être publics (comme 
les charter schools américaines) ou privés subventionnés par un programme de bons d'achat scolaires, les fameux "vouchers ", qui apportent aux parents une aide financière leur permettant de choisir une école privée (Chili, Colombie, Suède...). L'idée est ici d'évaluer l'impact du transfert des prérogatives de l'État central vers le local, quelle que soit la forme de cette dévolution ou les acteurs - publics ou privés qu'elle peut toucher. Nous verrons que la décentralisation est autant une affaire de transferts territoriaux de compétences que de transferts de pouvoirs politiques entre groupes sociaux.

\section{EXPANSION INTERNATIONALE DES POLITIQUES DE DÉCENTRALISATION DE L'ÉDUCATION}

Multiformes, les politiques nationales de décentralisation se sont multipliées durant les deux dernières décennies.

\section{La décentralisation s'est imposée dans des pays de niveaux de vie, de tailles et de structures politiques très variés}

La décentralisation s'est imposée dans des pays de niveaux de développement économique très divers, depuis des pays en voie de développement, comme le Kenya ou les Philippines et leur réseaux d'écoles communautaires, jusqu'à des pays industrialisés, comme les pays nordiques ou le Royaume-Uni, en passant par les pays en transition (Argentine, Pays de l'Est...).

La décentralisation a également touché de nombreux pays, quelle que soit la taille de leur territoire ou l'importance de leur population. Des États vastes, comme l'Argentine (" provincialisation » de son éducation), aussi bien que des petits territoires comme le Danemark (« municipalisation » de son éducation) ou le Salvador (programme d'écoles autonomes EDUCO) se sont convertis à la décentralisation. Des États peuplés, comme l'Espagne, ou de plus petits pays, comme le Salvador et son réseau d'écoles autonomes ont inscrit la gouvernance locale dans leur agenda.

Si la décentralisation a touché bien sûr en priorité les structures unitaires comme le Pakistan, les anciens pays de l'Est ou la Chine, les États fédéraux ont également multiplié les expériences d'autonomie scolaire (États-Unis, Australie, Inde...).
Tous les continents ont été concernés : depuis l'Afrique (Zimbabwe, Kenya, Zaïre...), en passant par l'Asie (Indonésie, Thaïlande, I'Inde...), l'Europe (anciens pays de l'Est, pays nordiques, Espagne, Portugal...), l'Amérique du Nord (États-Unis), ou le Pacifique (Nouvelle Zélande, Australie...) ... En Amérique Latine, quasiment tous les États ont mené des expériences de décentralisation.

On le voit donc, la décentralisation n'est pas une réponse ponctuelle à un niveau de développement économique ou à un stade d'expansion des systèmes éducatifs : elle est utilisée à la fois pour répondre à des problèmes d'expansion des structures éducatives ou comme solution à un souci de qualité de l'éducation dans les systèmes plus matures. Ce n'est pas non plus une solution apportée à des problèmes d'organisation territoriale ou démographique : elle s'impose dans des pays de taille et de population variées. Elle n'est pas non plus rattachée à une culture ou à une zone géographique.

\section{Les réformes de décentralisation répondent à des motivations très diverses}

Les réformes de décentralisation se sont imposées dans de nombreux pays parce qu'elles répondent à des objectifs variés.

Si la gouvernance locale apparaît aujourd'hui comme LA solution aux problèmes de qualité de l'éducation, la première vague de décentralisations, dans les années 70-80, s'explique plus particulièrement par des contingences financières et fiscales.

Le transfert de compétences éducatives vers les acteurs locaux est d'abord perçu comme une solution à la fois aux déficits budgétaires importants des États centraux et au souhait de poursuivre l'expansion des structures éducatives, malgré la contrainte financière (Winkler, 1989). C'est l'époque où les généraux argentins décentralisent l'éducation primaire vers des provinces qui refusent ce transfert, où le Kenya ou les Philippines développent leurs réseaux d'écoles communautaires censées attirer les subsides des familles.

Les années 90 sont davantage marquées par les décentralisations dites démocratiques, l'objectif est alors de couper avec un passé autoritaire symbolisé par une structure centralisée en transférant parfois vers des conseils d'écoles mais surtout vers les collectivités locales, des compétences, entre autres, dans le domaine de l'éducation. Après l'exemple emblématique de l'Espagne, dans les années 80, les 
anciens pays de l'Est mais aussi des pays d'Amérique Latine comme l'Uruguay suivent cette voie.

Outre la démocratisation qui s'accompagne le plus souvent d'un transfert vers les autorités politiques, la décentralisation commence à rimer dans les années 90 avec qualité de l'enseignement. La gouvernance locale prend alors le plus souvent la forme de l'autonomie scolaire (Winkler et Gersberg, 1998). Ces politiques ne touchent alors souvent que le domaine éducatif. C'est le cas au Nicaragua, au Salvador ou dans certains États brésiliens comme le Minas Gerais.

On voit donc que, parmi les motivations des politiques de décentralisation, l'amélioration de la qualité de l'enseignement n'apparaît que tardivement. Les réformes de décentralisation de l'éducation ont été moins des outils de politiques éducatives que des instruments au service d'objectifs étrangers à la qualité de l'enseignement.

Ces objectifs variés, potentiellement contradictoires, expliquent que les réformes de décentralisation soient inscrites sur les agendas de tous les partis politiques: gouvernements de droite comme en Angleterre sous le règne Tchatcher ; partis de gauche comme en NouvelleZélande; voire même gouvernements autoritaires comme Pinochet au Chili ou les généraux en Argentine. La décentralisation survit, de plus, aux remaniements de gouvernements : entamée par les partis de droite, elle est confirmée par la gauche, comme en Espagne; amorcée par la gauche elle est reprise à son compte par la droite comme en France, imposée par des régimes totalitaires, elle est confirmée par les gouvernements démocratiques comme au Chili ou en Argentine.

\section{Les répartitions de compétences entre gouvernements centraux et pouvoirs locaux varient fortement suivant les pays}

Les motivations diverses de la décentralisation expliquent qu'aucune réforme de gouvernance locale n'ait été totale. II n'existe pas aujourd'hui d'État complètement décentralisé en matière d'éducation : les organisations nationales se caractérisent davantage par un équilibre entre des fonctions détenues par le pouvoir central et des compétences déléguées à des unités locales. Le tableau ci-dessous montre que la répartition des compétences en éducation varie fortement suivant les pays.

Dans aucun pays, les collectivités locales ou les écoles ne détiennent, en toute autonomie, l'ensemble des pouvoirs. Même les États fédéraux connaissent aujourd'hui des règles nationales, sous forme de standards éducatifs (USA, Australie...), d'échelles de salaires nationales (Allemagne) ou de reconnais-

Tableau I. - Répartition des compétences pour certaines fonctions éducatives dans l'enseignement basique obligatoire, dans une sélection de pays (2003)

\begin{tabular}{|l|c|c|c|c|c|c|c|c|c|c|}
\hline \multicolumn{1}{|c|}{$\begin{array}{c}\text { Compétences/ } \\
\text { Pays }\end{array}$} & France & Japon & Luxembourg & Norvège & Espagne & Mexique & USA & Suède & UK & $\begin{array}{c}\text { Nouvelle } \\
\text { Zélande }\end{array}$ \\
\hline $\begin{array}{l}\text { Conception } \\
\text { programmes }\end{array}$ & 1 & 1 & 1 & 1 & 1 & 1 & 2 & 3 & 3 & 3 \\
\hline Certification & 1 & 1 & 1 & 1 & 2 & 2 & 2 & 1 & 1 & 1 \\
\hline $\begin{array}{l}\text { Recrutement } \\
\text { enseignants }\end{array}$ & 1 & 2 & 1 & 2 & 2 & 2 & 2 & 3 & 3 & 3 \\
\hline $\begin{array}{l}\text { Détermination } \\
\text { conditions de service } \\
\text { enseignants }\end{array}$ & 1 & 1 & 1 & 2 & 1 & 1 & 2 & 2 & 1 & 1 \\
\hline Allocation budgétaire & 1 & 2 & 1 & 2 & 2 & 2 & 2 & 2 & 3 & 3 \\
\hline
\end{tabular}

Source : auteur. Les pays sont classés en fonction d'une intervention croissante des acteurs locaux, collectivités locales et/ou établissements scolaires.

Légende :

1 = État central, 2 = Collectivités locales, 3 = établissements scolaires.

Acteur agissant en toute autonomie

Acteur prédominant dans l'exercice de la compétence mais agissant en collaboration avec d'autres centres de pouvoirs. 
sances mutuelles des diplômes (Suisse, Canada...). Les pays qui ont mené les politiques d'autonomie scolaire les plus volontaristes (Nouvelle-Zélande, Royaume-Uni, Hongrie...) encadrent de fait très rigoureusement le fonctionnement de leurs écoles, par des objectifs scolaires nationaux, par des inspections nombreuses ou par des règles salariales nationales.

Durant les années 80 et 90 , les compétences éducatives ont été diversement décentralisées.

Les transferts les plus massifs ont été réalisés dans le domaine de la gestion et du financement. Ils ont pris plusieurs formes. II s'agissait, en effet, soit de transférer massivement la responsabilité financière de l'expansion des structures éducatives vers les collectivités locales (Argentine...), soit d'autoriser les écoles à gérer leurs propres budgets afin de rationaliser l'allocation des ressources (Hongrie, NouvelleZélande...), soit, dans le cadre des "décentralisations démocratiques ", de permettre aux collectivités locales d'intervenir dans les affaires éducatives, mais en les cantonnant au financement et à la gestion de domaines restant en marge des activités pédagogiques, comme les équipements, les cantines ou l'aide sociale (France, Portugal, Grèce...).

Parce que l'on touche au cœur de l'activité pédagogique, les programmes scolaires ont été délégués avec beaucoup plus de parcimonie. II n'existe pas aujourd'hui de pays dans lequel les collectivités locales ou les établissements décident seuls des contenus enseignés. Dans le cadre des décentralisations démocratiques, les autorités locales se sont vu reconnaître le droit d'intervenir à la marge sous forme d'heures dédiées dans les programmes (pays nordiques, Indonésie...). Dans le cas d'États multiculturels, les programmes des provinces autonomes doivent intégrer une base nationale, les "minimos " en Espagne par exemple. Même les pays qui ont pratiqué la forme la plus radicale d'autonomie scolaire (Nouvelle-Zélande, Royaume-Uni) pilotent les activités pédagogiques de leurs écoles par une panoplie d'objectifs. Dans les pays fédéraux traditionnels comme les États-Unis ou l'Australie, on assiste même à une recentralisation des programmes scolaires par l'imposition de standards nationaux aux États.

Parce que l'on touche à un contre-pouvoir politique fort, la gestion du personnel a, plus encore que les programmes, été décentralisée avec précaution. Certes, les expériences les plus radicales ont transféré les recrutements vers les collectivités locales ou les écoles (Chili, Angleterre, Suède), mais il n'existe pas aujourd'hui de pays dans lequel les écoles décident librement des conditions de service et surtout des traitements des enseignants. Cette dernière compétence reste souvent l'apanage des gouvernements centraux, même dans certains États fédéraux (Allemagne...), et même dans les pays qui font largement participer les écoles à la gestion des enseignants (Belgique, Irlande, Nouvelle-Zélande...). Non seulement cette fonction n'a jamais vraiment été décentralisée mais elle a, au contraire, été recentralisée, dans le cadre de politiques de revalorisation de la profession, comme au Brésil. La gestion du personnel, enseignant ou non enseignant, est le dossier sensible de la décentralisation, comme l'a montré le cas français.

\section{TYPOLOGIE DES RÉPARTITIONS DE COMPÉTENCES EN ÉDUCATION}

Marquée donc à la fois par des forces centrifuges et centralisatrices, l'évolution récente de la répartition des compétences en éducation a fait voler en éclats la traditionnelle opposition qui existait entre les États centralisés et les États fédéraux. La typologie de la répartition des pouvoirs en éducation s'est ainsi enrichie de nouvelles familles.

\section{Le modèle de l'“ État centralisé » se craquelle}

Symbole du développement de l'instruction publique obligatoire depuis le $\mathrm{XIX}^{\mathrm{e}}$ siècle, le modèle de l'État centralisé voit le ministère de l'Éducation, et une administration déconcentrée plus ou moins autonome, définir les règles de fonctionnement dans tous les domaines : pédagogie (programmes centralisés, examens externes nationaux...), gestion des ressources humaines (recrutement national souvent sur concours, conditions de service négociées nationalement...), finances (financement national du système éducatif, affectation du budget et gestion des écoles par l'État central ou par des administrations déconcentrées gardées sous forte tutelle). L'État central est à la fois concepteur, opérateur et contrôleur du système éducatif. Sous la poussée des réformes de décentralisation entreprises quasi systématiquement dans tous les États centralisés, ce schéma monolithique s'est craquelé. La plupart de ces pays ont commencé à déléguer partiellement certaines fonctions à d'autres centres de pouvoirs, comme les collectivités territoriales. Mais, pour cette famille de 
pays, il s'agit pour l'instant de transferts de compétences restreints, principalement opérés dans le domaine de la gestion des équipements et/ou du primaire. C'est le cas en France, au Japon, au Portugal ou au Luxembourg. Ces timides décentralisations n'entament en rien le pouvoir décisionnaire de l'État central : les autorités locales se voient confier peu de compétences dans les domaines stratégiques de la pédagogie et de la gestion du personnel.

La régression du modèle centralisé a permis l'expansion de deux familles d'États nouvellement décentralisés.

\section{Le modèle de "collaboration État central-pouvoir local " s'impose dans les pays de culture sociale consensuelle}

Issu de réformes de décentralisation modérées, le modèle de "collaboration État central-pouvoir local " voit l'État central rester présent mais aussi laisser les pouvoirs locaux intervenir sur l'ensemble de ses compétences traditionnelles. C'est la fin de la politique de la chasse gardée, les autorités locales et les écoles se voient reconnaître des pouvoirs décisionnaires dans tous les domaines.

Elles participent, tout d'abord, à la définition des activités pédagogiques par le biais de mécanismes variés : quotas d'heures scolaires laissés à leur discrétion, options libres, possibilité d'augmenter les horaires des disciplines obligatoires en respectant un plafond maximum national... Pour autant, l'État central continue à encadrer rigoureusement les activités pédagogiques par la définition de disciplines obligatoires avec des horaires fixes. La marge de manœuvre des acteurs locaux est en général, également, limitée dans la gestion des ressources humaines. Leur pouvoir est, au contraire, beaucoup plus large dans le domaine du financement et de l'administration des écoles. Étant fortement sollicitées financièrement - dans ce schéma les collectivités locales contribuent au financement du système sur leurs propres deniers -, elles se voient attribuer le pouvoir de gestion - corollaire des obligations de financement. Les municipalités, provinces et autres régions - tous pouvoirs politiques autonomes -, se retrouvent ainsi en charge de l'administration de niveaux scolaires entiers, en général, le primaire et le secondaire général. Les pays nordiques, comme I'Islande, le Danemark, la Norvège sont l'archétype de cette famille également représentée par certains anciens États de l'Est (Pologne, République Tchèque...).

\section{Les révolutions des "États décentralisateurs- volontaristes "}

Plus révolutionnaire, la famille des «États décentralisateurs-volontaristes ", avec la Hongrie, la NouvelleZélande, le Royaume-Uni ou l'Espagne s'est étoffée dans les années 90 .

En conférant aux écoles ou aux pouvoirs politiques locaux des pouvoirs extraordinaires, en totale rupture avec les systèmes dont ils sont issus, cette troisième famille est née de réformes de décentralisation qui s'apparentent à de mini-révolutions. Des États fortement centralisés ont résolument tourné le dos à leur ancienne organisation et fait nettement le choix du local.

L'État central n'abdique pas tout pouvoir dans l'organisation du système éducatif : mais ses compétences se limitent à la régulation et au contrôle, l'ensemble des tâches de gestion sont déléguées aux acteurs locaux, le plus souvent aux écoles qui bénéficient d'un large statut d'autonomie. II s'agit de délégation de management au sens large. Les pouvoirs locaux, surtout les écoles, interviennent tout d'abord dans la conception des programmes : l'État central par la fixation d'objectifs ou de programmes obligatoires minimaux pilote le système par les résultats, à charge pour les écoles de définir dans le détail les programmes scolaires qui permettront de présenter, lors de tests standardisés nationaux ou d'inspections nationales nombreuses et détaillées, des performances à la hauteur de cette liberté déléguée. Les acteurs locaux sont également en charge de la gestion des ressources humaines : ils recrutent, licencient et plus rarement déterminent partiellement les salaires des enseignants. Ils définissent leur budget et peuvent recueillir des fonds localement. Le statut d'entité légale, souvent conféré aux écoles de ce modèle, leur permet une gestion quasi autonome.

\section{États fédéraux : un double mouvement de centralisation et d'autonomie scolaire}

Plus traditionnels, les États fédéraux (Canada, États-Unis, Brésil ou Allemagne) confèrent aux États, cantons ou autres provinces la responsabilité entière des systèmes éducatifs. C'est eux qui définissent le système (programme, certification, règles de recrutement, d'administration financière), qui le gèrent, souvent par le biais d'autorités locales comme les districts américains, et qui le contrôlent. Mais les années 90 ont vu ces systèmes éducatifs ancrés dans l'histoire évoluer dans deux directions a priori opposées : 
un mouvement de centralisation et le transfert de nouvelles compétences aux écoles. La centralisation de ces systèmes a été réalisée par deux mécanismes : 1) l'imposition de standards nationaux, d'abord facultatifs puis de plus en plus contraignants - dans les pays dominés par un État fédéral fort comme les États-Unis, le Brésil ou l'Australie ou 2) des initiatives de coopérations inter-provinces dans la création des programmes, les reconnaissances des diplômes... comme au Canada, en Suisse ou en Allemagne. À l'opposé, les nombreuses expériences d'autonomies des écoles aux USA, en Australie ou au Brésil, ont laissé davantage de marge aux acteurs locaux professionnels de l'éducation.

\section{LES THÉORIES DE LA DÉCENTRALISATION EN ÉDUCATION}

Si les réformes de décentralisation se sont multipliées, il ne semble pas qu'elles puissent trouver leur légitimité dans une argumentation théorique solide. Pour Ozler et King (1998), il n'existe pas de théories consistantes qui expliquent pourquoi et comment la gouvernance locale peut avoir des effets positifs sur les performances des élèves. Les fervents défenseurs de la thèse locale puisent souvent leurs arguments dans les théories générales de la décentralisation et extrapolent sur l'éducation.

\section{La défense de la décentralisation}

L'idée centrale des défenseurs de la décentralisation est que plus on rapproche la gestion scolaire des écoles, en transférant des compétences aux pouvoirs locaux (provinces, États, lander ou autres municipalités) voire aux unités scolaires elles-mêmes, meilleure sera la fourniture de services éducatifs (Winkler, 1989).

Et ce pour trois raisons principales, que nous énonçons très schématiquement ici.

En rapprochant les producteurs de service de l'utilisateur final, la gouvernance locale permet tout d'abord de produire des services plus en adéquation avec les besoins locaux (Oates, 1972). Les théoriciens ne s'accordent cependant pas sur les mécanismes mis en jeu. Pour certains, c'est une meilleure information des agents locaux (Musgrave, 1959), pour d'autres la crainte d'être sanctionné lors des élections locales, pour d'autres encore (Tiebout, 1956) ce serait la compétition entre les gouvernements subnationaux qui souhaitent garder leurs habitants et leurs taxes plutôt que de les voir " voter avec leurs pieds ", c'est-à-dire déménager s'ils ne trouvent pas la politique locale positive.

Dans cette logique, plus les délégations de pouvoirs se font auprès de l'unité scolaire, meilleurs sont les résultats. "Plus la décision est locale, plus la voix du consommateur-voteur peut se faire entendre. Cela signifie qu'elle est plus entendue au niveau de l'école qu'au niveau municipal, et de même qu'elle est plus efficace dans les structures de décisions strictement dédiées à l'éducation, comme les school districts, que dans les gouvernements locaux couvrant un vaste éventail de domaines ", expliquent Winkler et Gershberg (1999). En permettant des variations dans les services éducatifs distribués, voire même en autorisant une compétition entre les écoles, la décentralisation est censée renforcer les capacités d'innovation des établissements scolaires et donc des résultats scolaires des élèves (Hoxby, 2000). En permettant d'apporter une solution adaptée à chaque individu et non des services éducatifs homogènes, la décentralisation quand elle prend la forme de l'autonomie scolaire, permettrait d'offrir un enseignement personnalisé et même d'améliorer l'équité du système éducatif (Caldwell, 1990).

Second argument : la décentralisation est démocratique et participative. Quand elle prend la forme de l'autonomie scolaire, par exemple, elle permet une plus grande implication des enseignants dans les processus de décision, ce qui en retour accroîtrait leur motivation (Seddon, Angus et Poole,1990). La participation de la communauté est également censée enrichir la vie scolaire et accroître le contrôle sur les structures éducatives.

Troisième avantage de l'adoption de structures éducatives décentralisées : elles sont censées permettre une meilleure allocation des ressources éducatives (Murphy, 1991). La décentralisation est censée réduire les coûts de fonctionnement en allégeant la bureaucratie nationale (Raywid, 1990), en réduisant le temps de prise des décisions, en rendant plus efficace le contrôle des dépenses locales, en permettant aux écoles de trouver les meilleurs contractants aux meilleurs prix...Le management local plus proche des besoins locaux et mieux contrôlé par les utilisateurs finaux permettrait une meilleure utilisation des deniers publics. 
Autre avantage financier de la décentralisation : elle permettrait d'alléger les finances publiques en générant de nouvelles ressources privées (Litvack et Seddon, 1999) : en effet, les familles associées au fonctionnement des écoles seraient plus enclines à participer financièrement à l'éducation de leurs enfants.

\section{De multiples attaques contre la décentralisation}

À ces allégations, les détracteurs de la décentralisation opposent de multiples arguments (Prud'homme, 1995).

Ils combattent tout d'abord les prétendues qualités de la décentralisation. Faute d'économies d'échelle et du fait de la duplication des structures et des décisions, ils doutent que la décentralisation puisse conduire à une allocation plus efficace des ressources.

De même, si la gouvernance locale peut permettre un contrôle plus vigilant des décisionnaires locaux, elle pourrait également conduire à la capture du pouvoir local par certains groupes d'intérêt dont le pouvoir est accru localement (Bardhan et Mookherjee, 1998).

De même, la multiplicité des centres de décision peut entraîner des problèmes de recrutement : toutes les régions disposeront-elles des ressources humaines compétentes pour mettre en œuvre les nouvelles politiques éducatives dont elles ont désormais la charge?

Mais surtout les opposants de la décentralisation pointent du doigt les risques inhérents à la gouvernance locale.

Tout d'abord, la décentralisation ne tiendrait pas compte de l'existence d'externalités, c'est-à-dire du fait que l'éducation peut avoir pour l'ensemble de la société des retombées économiques et sociales supérieures aux avantages personnels qu'elle procure à l'individu qui en bénéficie. Or, même si le débat sur le sujet n'est pas encore clos, les externalités seraient fortes dans le domaine de l'éducation. D'après Lucas (1988), le rendement social de l'éducation, c'est-à-dire la somme des avantages que la société peut retirer de cet investissement, serait supérieur à son rendement privé. En permettant aux utilisateurs finaux de prendre des décisions uniquement en fonction de leurs intérêts individuels, la décentralisation nie le caractère global de l'éducation et peut conduire les contribuables à ne pas assez investir dans les services éducatifs.

Les opposants à la décentralisation pointent également du doigt les inégalités territoriales et sociales que de telles réformes peuvent générer. En laissant aux communautés (villes, régions, provinces...) le soin de financer les services éducatifs, souvent sur leurs deniers propres, ou en accroissant les pouvoirs de management et de gestion des écoles, la décentralisation peut entraîner de fortes variations dans les ressources éducatives. Ces dernières peuvent en effet varier en fonction de la richesse des communautés mais aussi de la priorité que ces dernières donnent à l'investissement éducatif (Winkler, 1989).

\section{LA RECHERCHE EMPIRIQUE NE LÉGITIME PAS À CE JOUR LES RÉFORMES DE DÉCENTRALISATION}

Pas plus que l'argumentation théorique, le débat empirique sur la décentralisation ne semble ni clos ni apporter une justification solide aux politiques de gouvernance locale en éducation.

La décentralisation est marquée en éducation par une littérature importante mais principalement descriptive. Les études portant sur l'impact pédagogique de la gouvernance locale ou sur ses mécanismes sont rares.

Ces recherches présentent, de plus, des conclusions discordantes.

Quelques résultats ressortent, cependant, dans certains domaines.

\section{L'assiduité des élèves semble renforcée par les réformes de décentralisation}

Tout d'abord, il semblerait que la gouvernance locale, surtout quand elle prend la forme de l'autonomie scolaire, puisse avoir des conséquences positives sur l'absentéisme des élèves, du fait d'une plus grande implication des parents et de l'ensemble de la communauté. Ainsi, au Brésil, la réforme d'autonomie scolaire (élection des directeurs et des conseils d'école, gestion par les écoles des ressources financières...) initiée dans l'État du Minas Gerais et étendue dans d'autres États, s'est traduite par une réduction de l'absentéisme (Paes de Barros et Mendonça, 1998). Les mêmes résultats ont été constatés dans 
l'expérience d'autonomie des écoles à Chicago. D'après Pandey (2000), les États indiens ayant mis en œuvre, depuis 1994, le District Primary Education Program (DPEP), qui associe étroitement les communautés au développement scolaire, ont connu une rapide expansion de la scolarisation, en particulier de celle des filles.

\section{La décentralisation serait associée à une réduction de l'absentéisme des enseignants}

De la même façon, le contrôle exercé sur les enseignants semble avoir eu dans certaines expériences des effets positifs sur leur absentéisme. En Inde, d'après Pandey (2000), l'assiduité des enseignants a fortement progressé depuis que les Comités Villageois d'Éducation en sont responsables. King et Ozler (1998) rapportent qu'au Nicaragua les écoles qui bénéficient d'un statut d'autonomie et d'une plus grande participation des parents, connaissent également un taux d'absentéisme enseignant moins important. De même, au Salvador, les écoles autonomes EDUCO, qui sélectionnent elles-mêmes leurs propres enseignants, connaissent moins d'absences.

Si la décentralisation semble avoir un effet positif sur l'absentéisme des élèves et des enseignants, paradoxalement, elle ne semble pas induire automatiquement une progression des résultats académiques.

\section{L'association entre décentralisation et performances académiques reste à démontrer}

Certes, récemment, certaines évaluations ont montré un lien positif entre gouvernance locale et résultats scolaires. Ainsi, en Argentine, Eskeland et Filmer (2002) ont trouvé que l'autonomie des établissements scolaires et la participation des parents sont associés à des résultats supérieurs des élèves aux évaluations des $6^{\mathrm{e}}$ et $7^{\mathrm{e}}$ années de l'enseignement de base. De même, au Nicaragua, (Ozler et King 1998), le degré d'autonomie des écoles, surtout dans le domaine de la gestion des enseignants, est corrélé positivement avec le niveau académique des élèves. Par contre, le simple statut d'autonomie ne suffit pas pour qu'une école améliore ses performances scolaires. King et Ozler font là une distinction importante entre l'autonomie réelle et l'autonomie légale. La réforme radicale anglaise de 1988, caractérisée par le choix parental et une forte autonomie des établissements scolaires, se serait traduite par des résultats en hausse à l'examen de fin de secondaire (Gorard et
Taylor, 2002). Les politiques d'autonomie scolaire menées depuis les années 80 aux États-Unis, comme celle de Chicago (Bryk, Thum, Easton, et Luppescu, 1998) ont été associées, dans le temps, également à une progression des résultats scolaires des élèves.

Au total, on voit là que, exception faite de l'Argentine, les politiques de décentralisation qui ont eu des répercussions positives sur les performances des élèves sont davantage orientées sur l'autonomie scolaire que sur le transfert de compétences aux collectivités locales. L'exemple du Chili est encore plus probant. Alors que la réforme des années 80 , fortement axée sur la municipalisation des services éducatifs et l'introduction de la compétition et du choix parental, ne semble pas avoir eu d'impact sur les résultats académiques (McEwan et Carnoy, 1998), la seconde phase de réforme entamée en 1990 et plus axée sur l'établissement scolaire a vu les résultats des élèves au test standardisé, le SIMCE, fortement progresser (Cox et Lemaitre, 1999).

À ces exemples positifs, s'opposent un grand nombre de cas où les réformes décentralisatrices n'ont pas eu d'impacts positifs sur les résultats académiques des élèves. Ainsi, au Brésil, la réforme menée dans le Minas Gerais, a conduit Paes de Barros et Mendonça (1998) à conclure que l'établissement de conseils d'école et les transferts financiers directs aux établissements n'étaient pas associés à une progression des résultats académiques. De même au Salvador, les performances des écoles autonomes EDUCO sont identiques à celles des établissements traditionnels (Jimenez et Sawada, 1998). Aux Philippines, le programme d'écoles communautaires n'a pas permis également d'améliorer la qualité de l'éducation (Lockeed et Zhao, 1992).

Les comparaisons internationales abondent dans le même sens : Meuret, Prod'hom et Stocker (1995) n'ont trouvé aucun lien entre le degré d'autonomie des collèges et le niveau des élèves en lecture à 14 ans, mesuré par l'enquête internationale Reading Literacy (1990). Globalement les revues de la littérature sur le sujet (Duru-Bellat et Meuret (2001), Leithwood et Menzies (1998) ...), surtout axées sur l'autonomie scolaire, concluent toutes au même résultat : les réformes de décentralisation dans l'éducation ne conduisent pas automatiquement à une amélioration de la qualité de l'enseignement.

Les explications avancées sont multiples et certainement complémentaires. 
Les réformes de décentralisation n'auraient pas des effets immédiats sur les acquisitions des élèves : un délai de cinq à dix ans serait nécessaire pour que ces réformes de structures affectent les apprentissages. Les expériences américaines à Chicago et à Memphis semblent, par exemple, aller dans ce sens-là.

Seconde explication : instrument légal, la décentralisation n'est pas toujours mise en œuvre par les acteurs du système. C'est la fameuse " empty opportunity » de Lockheed et Zhao (1992) : « la décentralisation ne produit pas à elle seule un contrôle local des écoles ». Le décalage entre la légalité et la réalité des réformes explique par exemple qu'au Nicaragua, ce ne soit pas le statut d'autonomie des écoles qui est associé aux résultats des élèves mais le pourcentage de décisions réellement prises localement (Ozler et King, 1998).

Autre explication : lorsque les décentralisations ne restent pas lettres mortes, il semblerait que leurs effets pédagogiques soient limités parce que, tout simplement, en modifiant les structures et non les méthodes pédagogiques, elles ne changent pas les variables qui affectent réellement les apprentissages des élèves (Leithwood et Menzies, 1998). Permettre aux enseignants d'intervenir davantage dans la vie de l'école ne conduit pas nécessairement à un changement dans leurs méthodes d'enseignement. De plus, les conseils d'écoles qui ne sont pas exclusivement constitués de professionnels de l'éducation se préoccupent rarement des questions liées aux contenus scolaires et aux méthodes d'apprentissage (Thrupp, Harold, Mansell et Hawksworth, 2000). Globalement, toutes les recherches concordent sur le fait que la décentralisation, même lorsqu'elle prend la forme de l'autonomie scolaire, ne conduit pas aux innovations pédagogiques tant espérées par les théoriciens de la gouvernance locale. Et Leithwood et Menzies de conclure : «des améliorations significatives des apprentissages scolaires ne peuvent être obtenues qu'avec la mise en œuvre généralisée de méthodes d'apprentissages plus efficaces". D'après eux, il semble qu'il existe donc des solutions plus directes, plus rapides et moins onéreuses pour réformer l'école.

Wössmann (2000) ainsi que Duru-Bellat et Suchaut (2004) apportent une autre explication aux faibles résultats des politiques de décentralisation. D'après eux, pour être efficaces, les systèmes éducatifs ne doivent pas être totalement décentralisés : un équilibre doit être trouvé entre des compétences qui doivent rester du ressort du gouvernement central (les programmes scolaires, le contrôle de la qualité de l'enseignement par des certifications nationales externes...) tandis que d'autres fonctions, comme la gestion des ressources ou les méthodes pédagogiques, doivent être transférées aux acteurs locaux. La solution ne passerait pas donc par une gouvernance locale totale.

\section{Les décentralisations sont potentiellement porteuses d'inégalités scolaires}

Si les études analysant l'impact de la décentralisation sur les résultats scolaires produisent des résultats fort divergents, les chercheurs s'accordent sur un point : la gouvernance locale est potentiellement porteuse d'inégalités territoriales et sociales.

En Chine, West et Wong (1995) ont ainsi montré que la politique de décentralisation avait entraîné l'apparition de disparités dans la fourniture de services éducatifs entre les régions. De même, Winkler et Rounds (1996) ont démontré que la politique chilienne de gouvernance locale avait conduit à des disparités dans les dépenses d'éducation. De la même façon, en Hongrie, le transfert aux municipalités de la gestion et d'une partie du financement des écoles secondaires a conduit à des différences de performances scolaires entre les villes de tailles différentes (Lockheed, 1996). En Argentine et au Brésil, il semblerait que la dispersion territoriale dans la qualité des services, mesurée à travers des indicateurs intermédiaires, comme les salaires ou le niveau de formation initiale des enseignants, se soit accrue après les réformes de décentralisation (Di Gropello, 1999).

II semble que globalement les programmes de vouchers n'aient pas atteint leur double objectif d'excellence scolaire et d'amélioration de l'équité des systèmes éducatifs. Certes, ces programmes combinent une double politique de "décentralisation-privatisation " et de choix scolaire, rendant difficile leur évaluation. Cependant, leurs résultats divergent selon les pays analysés. Angrist et al. (2001) ont trouvé qu'en Colombie le programme de vouchers avait permis d'accroître la scolarisation des enfants des milieux défavorisés à un moindre coût. Mais les difficultés méthodologiques sont importantes : Gaury (1998) a montré qu'au Chili, les résultats des écoles privées, subventionnées par un programme de voucher, étaient supérieurs parce qu'elles avaient recruté des élèves présentant un meilleur potentiel académique. Dans cette lignée, plusieurs recherches ont conclu que les politiques de vouchers ne semblent pas améliorer les services éducatifs pour les enfants des milieux sociaux 
et territoriaux les plus défavorisés. Les évaluations des performances des charter schools américaines conduisent également à des résultats divergents.

\section{CONCLUSION}

Si durant les deux dernières décennies, la décentralisation de l'éducation est devenue l'une des politiques éducatives les plus populaires sur l'ensemble des continents, le débat théorique et empirique sur le sujet n'est à ce jour pas clos. Tant les mécanismes par lesquels la gouvernance locale est censée améliorer les performances scolaires que des justifications empiriques solides sont encore à apporter. Au total, il reste donc encore à démontrer que le recul de l'État central au profit d'acteurs locaux améliore l'efficacité et l'équité des systèmes éducatifs et ce quelle que soit la forme que prend ce désengagement : décentralisation territoriale, autonomie scolaire, programmes de privatisation par les vouchers... En mul- tipliant les points de vue sur des réformes encore trop souvent analysées dans leurs frontières nationales, les comparaisons internationales, peuvent nous aider à avancer dans l'analyse de l'impact de ces politiques. Certes, leur maniement n'est pas toujours facile : le même terme ne désigne pas toujours le même concept dans chaque langue, le décalage entre la réalité et la légalité des réformes peut varier entre les États, des contextes nationaux différents peuvent, au-delà des effets prévisibles que l'on souhaite analyser, entraîner des effets pervers particuliers... Cependant, ce n'est qu'en comparant les mises en œuvre et les effets des politiques de décentralisations dans plusieurs pays que la recherche pourra distinguer ce qui tient aux contextes nationaux et ce qui est inhérent au processus décentralisateur.

Nathalie Mons IREDU/CNRS

\section{BIBLIOGRAPHIE}

ANGRIST J., BETTINGER E., BLOOM E., KING E., KREMER M. (2001). - Vouchers for Private Schooling in Colombia : Evidence from a Randomized Natural Experiment. NBER Working Paper $n^{\circ}$ 8343, Cambridge, Mass.

BENNET R.J. (1990). - Decentralization, Local Governments and Markets. Towards Post-Welfare Agenda. Oxford, UK : Clarendon Press.

BARDHAN P., MOOKHERJEE D. (1998). - Expenditure Decentralization and The Delivery of Public Services in Developing countries. CIDER, Working Paper C98/104. Boston University.

BRYK A.S., THUM Y.M., EASTON J.Q., LUPPESCU S. (1998). - Examining Productivity : Ten-Years Trends in the Chicago Public Schools. Chicago : Consortium on Chicago School Resarch.

CALDWELL B. (1990). - School-Based Decision Making and Management : International Developments. In J. Chapman (eds.), School Based Decision Making and Management, p. 3-26. London, UK : Falmer Press.

COX C., LEMAITRE M.J. (1999). - Market and State Principles of Reform in Chile Education : Policies and Results. In D. Leiptziger and G. Perry (eds.), Chile : Recent Policy Lessons and Emerging Challenges. WDI Development Studies Series. Washington D.C. : World Bank.

DI GROPELLO E. (1999). - Educational Decentralization models in Latin America. Cepal Review, $n^{\circ} 68$, p. 155173.
DURU-BELLAT M., MEURET D. (2001). - Nouvelles formes de régulation dans les systèmes éducatifs étrangers : autonomie et choix des établissements scolaires. Revue Française de Pédagogie, $n^{\circ} 135$, p. 173-221.

DURU-BELLAT M., MONS N., SUCHAUT B. (2004). - Caractéristiques des systèmes éducatifs et compétences des jeunes de 15 ans : l'éclairage des comparaisons entre pays. Rapport pour le Ministère de l'Education. Dijon : Iredu.

ESKELAND G.S., FILMER D. (2002). - Autonomy, Participation, and Learning in Argentine Schools. Finding and their Implications for Decentralization. Policy Research Working Paper, WPS $n^{\circ} 2766$, Washington D.C. : World Bank.

GAURI V. (1998) - School Choice in Chile : Two Decades of Educational Reform. Pittsburgh, PA. : University of Pittsburgh Press.

GORARD S., TAYLOR C. (2002). - Market Forces and Standards in Education : a Preliminary Consideration. Bristish Journal of Sociology of Education, 23, 1, p. 5-18.

HOXBY C. (2000). - Does competition among public schools benefit students and taxpayers? American Economic Review, 90, 5, p. 1209-38.

JIMENEZ E., SAWADA Y. (1998). - Do Community-managed schools work? An Evaluation of El Salvador's EDUCO Program. World Bank Economic Review, 13, 3, p. 415-441.

KING E., ÖZLER B. (1998). - What's Decentralization Got To Do with Learning ? The Case of the Nicaragua's 
school autonomy reform. Development Economics Research Group, Working Paper series on Impact Evaluation of Education reforms, $n^{\circ} 9$, June, Washington, D.C. : World Bank.

LEITHWOOD K., MENZIES T. (1998). - Forms and Effects of School-based Management : A Review. Educational Policy, 12, 3, p. 325-346.

LITVACK J, SEDDON J. (1999). - Decentralization briefing notes. World Bank Institute Working Paper, Washington, D.C. : World Bank.

LOCKEED M., ZHAO Q. (1992). - The Empty Opportunity : Local Control of Secondary Schools and Student Achievement in the Philippines. Working Paper WPS 825, Washington DC : World Bank.

LUCAS R. (1988). - On The Mechanics of Economic Development. Journal of Monetary Economics, 22, 1, p. 342.

McEWAN P., CARNOY M. (1998). - Public investments or private schools ? A Reconstruction of Educational Improvements in Chile, processed, Washington, D.C. : World Bank

MEURET D., PROD'HOM J., STOCKER E. (1995). - Comparer les structures de décision des systèmes éducatifs : un bilan de l'approche quantitative. In OCDE-CERI, Mesurer la qualité des établissements scolaires (p. 33-58). Paris : OCDE-CERI.

MURPHY, J. (1991). - Restructuring Schools : Capturing and Assessing the Phenomena. New York: Teachers College Press, Columbia University.

MUSGRAVE R.A. (1959). - The Theory of Public Finance. New York : Mc Graw Hill.

OATES W. (1972). - Fiscal Federalism. New York : Harcourt Brace.

PAES DE BARROS R., SILVA PINTO DE MENDONÇA S. (1998). - The Impact of Three institutional Innovations in Brazilian Education. In W. D. Savedoff (eds.), Organisation Matters : Agency problems in Health and Education. Washington D.C. : Inter-American Development Bank.

PANDEY R. (2000). - Getting to Scale : The Story of DPEP in India. A presentation made as part of The Education Ministers Series, Education Reform and Management Group. Washington D.C. : World Bank.
PRUD'HOMME R. (1995). - The Dangers of Decentralization. The World Bank Research Observer, 10, 2, p. 201220.

RAYWID M.A. (1990). - Rethinking School Governance. In R.F. Elmore (Eds.), Restructuring Schools : The Next Generation of School Reform, p. 152-206. San Francisco CA : Jossey-Bass Inc.

RHOTEN D. (2000). - Education Decentralization in Argentina : a "Global-Local Conditions of Possibility" Approach to State, Market and Society Change. Journal of Education Policy, Vol. 15, 6, p. 593-619

RONDINELLI D., NELLIS J., CHEEMA G. S. (1984). - Decentralization in Developing Countries. World Bank Staff Working Paper No. 581, Washington, D.C. : World Bank.

SEDDON T., ANGUS L., POOLE M. (1990). - Pressures on the Move to School Based Management. In J. Chapman (Eds.), School Based Decision Making and Management, p. 29-54. London, UK : Falmer Press.

TIEBOUT C. (1956). - A pure Theory of Local Expenditures. Journal of Political Economy, 64, p. 416-424

THRUPP M., HAROLD B., MANSELL H., HAWKSWORTH L. (2000). - Mapping the Cumulative Impact of Education Reform : Final Report. New Zealand : University of Waikato.

WINKLER D. (1989). - Decentralization in Education : An Economic Perspective, Population and Human Resources Department, Working Paper WPS 143, Washington, D.C. : World Bank.

WINKLER D., ROUNDS T. (1996). - Municipal and Private Sector Response to Decentralization and School Choice. Economics of Education Review, 15, 4, p. 365-376

WINKLER D., GERSHBERG I. (1999). - Education Decentralization in Latin America : The Effects on the Quality of Schooling. LCSHD Paper Series $n^{\circ} 59$. Washington, D.C. : World Bank.

WEST L., WONG C. (1995). - Fiscal Decentralization and Growing Disparities in Rural China : Some Evidence in the Provision of Social Services. Oxford Review of Economic Policy, 11, 4, p. 70-84

WÖSSMANN L. (2000). - Schooling Resources, Educational Institutions, and Student Performance : The International Evidence. Kiel Working Paper $n^{\circ} 983$. Germany : KIWE. 